\title{
Evaluating Community Based Initiatives' Sustainability in Europe: Balancing Data Needs and Resulting Uncertainties.
}

\author{
Federico Martellozzo ${ }^{1}$, Dominik Reusser ${ }^{2}$, Helge Groß ${ }^{2}$
}

\begin{abstract}
:
Global environmental-change is evident, and undeniably it is mostly induced by anthropogenic activities. Several programs fuelling climate-change mitigation were lately implemented; all fostering specific and ambitious targets. Although some improvements were regionally observed, regrettably the expected results are in many cases still out of reach. Contemporaneously, societies experienced a proliferation of grass-roots initiatives calling for individual participation in fostering societal sustainable transition. Scholars advocate that bottom-up activities may outperform top-down policies in reaching sustainability; however, a methodological framework to intelligibly assess CBIs' impact on socio-ecological systems is still unexplored. This paper aims at: illustrating main caveats in assessing CBIs' environmental impacts, proposing a general methodological framework, presenting results from a pan-European research
\end{abstract}

Keywords: Environmental assessment; greenhouse gas emissions; community based intiatives; sustainable transition, sustainability, carbon footprint.

\section{Introduction}

The active participation in CBIs is a spreading phenomenon that has reached a significant magnitude (Conrad \& Hilchey 2011). In some cases Community Based Initiatives (henceforth CBIs) are also supposed to have catalyzed social and technological innovation, thus contributing to the societal transition into low-carbon economy (Seyfang \& Haxeltine 2012). Very often, CBIs are grass-roots initiatives with broad sustainability foci that promote a plethora of activities such as alternative transportation, urban gardening, renewable energy implementation, waste regeneration/reduction, etc. (Warner 2002; Wilson 2010). Some advocate that such practices fostered by bottom-up activities, rather than top-down policies, represent a proficient countermeasure to alleviate global environmental change and effectively foster a more permanent societal transition towards sustainability (While et al. 2010; Ostrom 1990; Takeuchi 2010). However, thus far most empirical research grounds mainly on anecdotal evidence (Meadowcroft 2011; O'Riordan 2004; Sultana 2009) and little work has been done to quantitatively assess CBIs" “environmental impacts" (EI) or their carbon footprints using comparative methodologies ( $\mathrm{Li}$ et al. 2013; Roseland 2000; Yang et al. 2010). Few studies are focusing on one specific aspect of the environment, such as energy (Brown 2004),

${ }^{1}$ University of Rome - La Sapienza. Dept. of Methods and Models for Territory Economics and Finance; via del Castro Laurenziano 9; 00161 Roma; Italy. Tel: (+39) 3429977690

2Potsdam Institute for Climate Impact Research. PIK; Telegraphenberg A 31; 14473 Potsdam; Germany.

*Corresponding author. 
carbon emissions (Kennedy \& Sgouridis 2011) or water (Galli et al. 2012; Tanner 2007). Environmental indicators developed to assess community level initiatives' impacts have been summarized in a section on natural science perspective studies on eco-village research (Marcus \& Wagner n.d., pp.89). The global eco-village network has developed a bottom-up Community Sustainability Assessment (CAS) based on a questionnaire that includes a comprehensive section on environmental aspects (Network 2001). Forrest (2011) bases his sustainability appraisal of small communities in transition on core generic criteria for sustainability assessment by Gibson in 2006 (Gibson 2006). Church (Church \& Elster 2002) bases his work on an assessment of environmental impacts at multiple scales (local community, local authority and national) and derives lessons for national policy. In all cases, the assessment is based on self-evaluation rather than measurable quantities. On the other hand, studies that evaluate a broad set of environmental indicators are often based on the ecological footprint methodology (Wackernagel \& Rees 1996). This method can be considered a top-down approach (Tinsley \& George 2006). The ecological footprint has not been without criticism because of the high level of aggregation, domination by energy consumption, a missing distinction between sustainable and unsustainable land use, the arbitrary selection of spatial scales to which the footprint is applied and a poor treatment of trade.

This research mainly aims at framing a methodology suitable to univocally assess CBIs' EI, which are crucial to the comprehension of CBIs' role in fostering sustainable transition (Seyfang \& Haxeltine 2012; Thakadu 2005). However, to this end, three main caveats need to be addressed (Tab. 1): first, some CBIs do not directly produce tangible measurable outputs, nor have an intelligibly defined set of inputs (e.g. the CBIs that focus on environmental education and dissemination). Thus, calculating their "indirect" EI may represent an intricate puzzle that is very much open to subjective interpretation. Second, CBIs' practices are heterogeneous and therefore existing methodologies to make comparisons of their EIs are neither straightforward nor proficient, also given the lack of available data. Third, another issue closely related to the one previously mentioned is the general lack of consensus among existing impact-assessment frameworks for certain practices (Galan et al. 2007; Mohareb et al. 2011; Pachauri \& Reisinger 2007; Peigné \& Girardin 2004; Saer et al. 2013).

These problems are harshened by the inhomogeneity of assumptions used to build several GHG frameworks, consequently resulting in a lack of reasonable baselines for GHG accounting protocols (Möllersten \& Grönkvist 2007). For instance, Chavez and his colleague advocate (Chavez \& Ramaswami 2011) that the first obstacle to be resolved while investigating EI based on carbon emission in order to support public intervention is to provide "... consistent, reproducible, comparable and holistic GHG framework that incorporate in-boundary and (possibly) trans-boundary GHG impacts of urban activities...". In this regard, this paper first frames the obstacles to be overcome in conceptualizing a meaningful EI assessment. Second, examples of boundaries and counterfactuals for the most relevant activities are discussed; these examples aim at covering all the domains in which the CBIs mapped within the TESS project operate (see Acknowledgements); hence although not exhaustive of all the activities done by CBIs in Europe, certainly the cases discussed are representative and include most of the CBIs populating the TESS inventory; besides, the domains (sectors) considered are 
responsible for more than $80 \%$ of global GHG emissions in the EU (European Environmental Agency 2013). Third, an original EI indicator for CBI based on a Comparative Carbon Accounting methodology is proposed and tested on few cases. These cases are taken from the inventory of CBIs that belong to the database of the TESS-Transition project (www.tess-transition.eu), which covers four main domains: food, energy, transport and waste. Preliminary results are presented and discussed. While we are aware that several caveats still need to be further explored and addressed, this novel application of a comparative methodology offers much to the existing literature on CBIs' impact assessment.

\section{Theoretical framework}

Finding a suitable index to assess univocally CBIs' EI is not an easy task for three main caveats, which are elicited and described below (Tab. 1); these are: unclear input/output, heterogeneity of activities, controversial practices.

Table 1. List of the main caveats regarding the development of a unique CBI's Environmental Impact Assessment.

\begin{tabular}{ll}
\hline Caveat & Description \\
\hline $\begin{array}{l}\text { unclear } \\
\text { input/output }\end{array}$ & $\begin{array}{l}\text { - Some CBIs do not produce a very clear output nor have a well-defined set of } \\
\text { input - this is the case of educational CBIs - hence, not solely calculating the } \\
\text { ensemble of secondary EIs may represent an intricate puzzle, but it is also } \\
\text { very much open to subjective interpretation of what are these EI. }\end{array}$ \\
heterogeneity of & $\begin{array}{l}\text { - CBIs' practices are very much heterogenic and it is not straightforward how } \\
\text { to proficiently compare their impact/footprint/effect on the environment. }\end{array}$ \\
controversial & $\begin{array}{l}\text { - Even if we disposed of a unique mean to calculate EI - although for certain } \\
\text { practices we have some reasonable options - for some practices the state of } \\
\text { the art does not offer a universally shared consensus about the environmental } \\
\text { evaluation of these practices (i.e. composting) }\end{array}$ \\
\hline
\end{tabular}

Hence any approximation on this subject is bound to sway between generalizations that are justified and simplifications that can be misleading; therefore, while elaborating the EIA methodology we have to aim at finding a set of general assumptions that may reasonably grant an appropriate equilibrium (Finnveden \& Moberg 2005).

\subsection{Unclear input/output and mixed objectives}

While the first caveat (Tab. 1) cannot be resolved completely, we can minimize subjectivity and ill-defined assessments with the use of a precisely defined framework. There are two basic options: (1) assessment of the full environmental footprint and (2) a comparative assessment against a counterfactual.

(1) A prominent example of in-depth environmental footprint assessment is called Life Cycle Assessment (LCA). It is based on the idea to potentially account for all the possible sources/causes of EI. In other words, the objective of the LCA is to establish a complete overview of the interactions with the environment of a product or service, helping to understand the environmental consequences directly or indirectly caused by its 
production and usage (Tukker 2000). LCA applications usually account for all possible influences on the environment, such the ones imputable to inputs, processing system, and interaction with the environment, so to assess thoroughly the EI potential reduction deriving from a specific CBI's activity (Manuilova et al. 2009). However, this is a very complex and time consuming task which requires a massive data gathering. Hence, it cannot be considered suitable for a method aiming at being general and widely applicable, but it may be the object of in depth analysis for specific cases.

(2) A method based on the "comparative" assessment with a counterfactual can be considered as a valid alternative. In this case the aim is to evaluate the EI per unit of output of each activity (e.g. the amount of energy used for its production in KWh, and the corresponding amount of GHG in $\mathrm{CO}_{2}$ equivalent) and compare it with the EI per unit of output of a standard counterfactual that is used as a baseline (e.g. see the GHG protocol for project accounting. Daviet \& Ranganathan 2005).

Using a baseline as a term of comparison opens up the possibility to eliminate specific units and hence could be reasonably used for inter-CBI comparison, disregarding its prevalent domain or activity. In this case, the choices of the counterfactual and of the boundaries are fundamental; consequently for each activity we must first decide what the main output is, which is not always straight forward.

\subsection{Heterogeneous activities}

The difficulty of selecting a main output is tightly linked to the second caveat described in table 1, the heterogeneity of CBIs. It may be further highlighted with the example of the so-called Solidarity Purchasing Groups (SPG). SPG's activity consists in replacing middleman so to connect local producers with a group of consumers. The main aim is to provide the purchasing group with local (often organic) food at a cheaper price (Cembalo et al. 2013). Thus, the main direct effect is to make beneficiaries saving money, but this is not strictly relevant for an evaluation of the SPG's EI; hence we shall solely focus on the carbon impact imputable to the delivery of that food. Hence, in spite of the fact that the activity deals with food its EI should be assessed as a transport activity (see paragraphs 3.1 and 3.3). To address this issue, we discuss and propose an EIA framework for a subset of activities that are the most common among the activities carried out by the CBIs populating TESS-Transition project inventory (Tab. 2). The options presented and discussed in Methods and Data cover the major fraction of typical activities in our inventory, however the examples presented in paragraphs 4.1, 4.2 and 4.3 aim at being representative but not exhaustive of the panorama of activities developed by CBIs, thus the methods proposed are meant to be extended and ad hoc applications to be developed for each activity making use of the same assessment principles. Nevertheless, single CBIs may then be described as one single activity of a combination of activities from this inventory.

\subsection{Controversial practices}

The third issue deals with the fact that some activities may have different description associated, thus generating diverse - and sometime controversial interpretations about the potential EI of the CBIs. For example, about debated activities as composting or aero/hydroponic agriculture literature does not offer a clear picture of 
which option is better, hence finding a reasonable counterfactual is even more critical because it obliges the analyst to take a stand. However, for the purpose of this exercise, we do not have to investigate if the option investigated is environmental friendly, rather to focus on assessing the environmental benefit (if any) coming from each CBI's activity given the respective boundaries when compared to a standard counterfactual. Besides, this issue is common to almost all GHG accounting framework aiming at being widely applicable; hence, particular care should be put for the choice of counterfactual and boundaries. Those factors are once more very important, because on these implicitly relies the capability of granting meaningful results while avoiding misleading simplifications.

\subsection{Basic steps of the assessment}

A robust assessment framework that leads to meaningful results is the GHG protocol for project accounting (Daviet \& Ranganathan 2005), although it is not very simple. The framework we propose in this study is well consistent with the GHG protocol for project accounting but its simplicity is enhanced. The GHG Protocol for Project Accounting identifies 6 basic steps in calculating a footprint. Step 1 regards the definition of the goal and scope of the assessment. Key is the identification of specific activities, the product or services these activities offer and their primary and secondary impacts on GHG emissions. The subsequent steps are repeated for all activities. The next three steps are about defining the counterfactual or baseline. The guide offers two options to estimate the baseline. In step 2, the procedure needs to be selected before performing the next steps. In step 3, both methods rely on assembling a set of baseline candidates for a certain region and time frame, by reflecting on the question how the same service would be offered in absence of the CBI. For the estimation of the baseline within step 4 , the first option to define the baseline is the project-specific procedure. It allows mainly semi-quantitative and qualitative evaluation of the different baseline candidates, making a selection based on the identification of barriers for each candidate and the conservativeness-principle. This procedure needs to be repeated for each CBI. The second option calculates a performance standard from the set of baseline candidates. It requires more data compared to the project-specific procedure. The performance standard can then be used for all CBIs engaging in a certain activity in this region. In step 5, data is monitored and GHG reductions are quantified. In step 6, the assessment is documented in a report, integrating the information about all activities. To assess the EI of CBIs, some premises complementing existing frameworks are necessary:

(1) Educational CBIs confront us with a very elusive output, thus, they do not fit into the EIA presented here.

(2) In order to keep the assessment simple (and most of all doable) for all CBIs the boundaries should be set as close as possible to the final product of their activity/procedure/practice.

(3) To make a potential EIA that can be compared across different activities we must rely on a measure that is general and not specific to any measure of unit. This can be achieved through a simple comparison between the EI of each activity and the EI of the corresponding counterfactual, or also through more statistically sophisticated elaboration and manipulation. 
Thus, this work aim at proposing a possible common approach to investigate the direct EI of CBIs under the premises of simplicity and cross-subdomain comparability described at point (ii) and (iii) above (Fig. 1). Besides, although direct EI of educational CBIs might be negligible (and could possibly be ignored) indirect impacts of dissemination activities should not be neglected when possible.

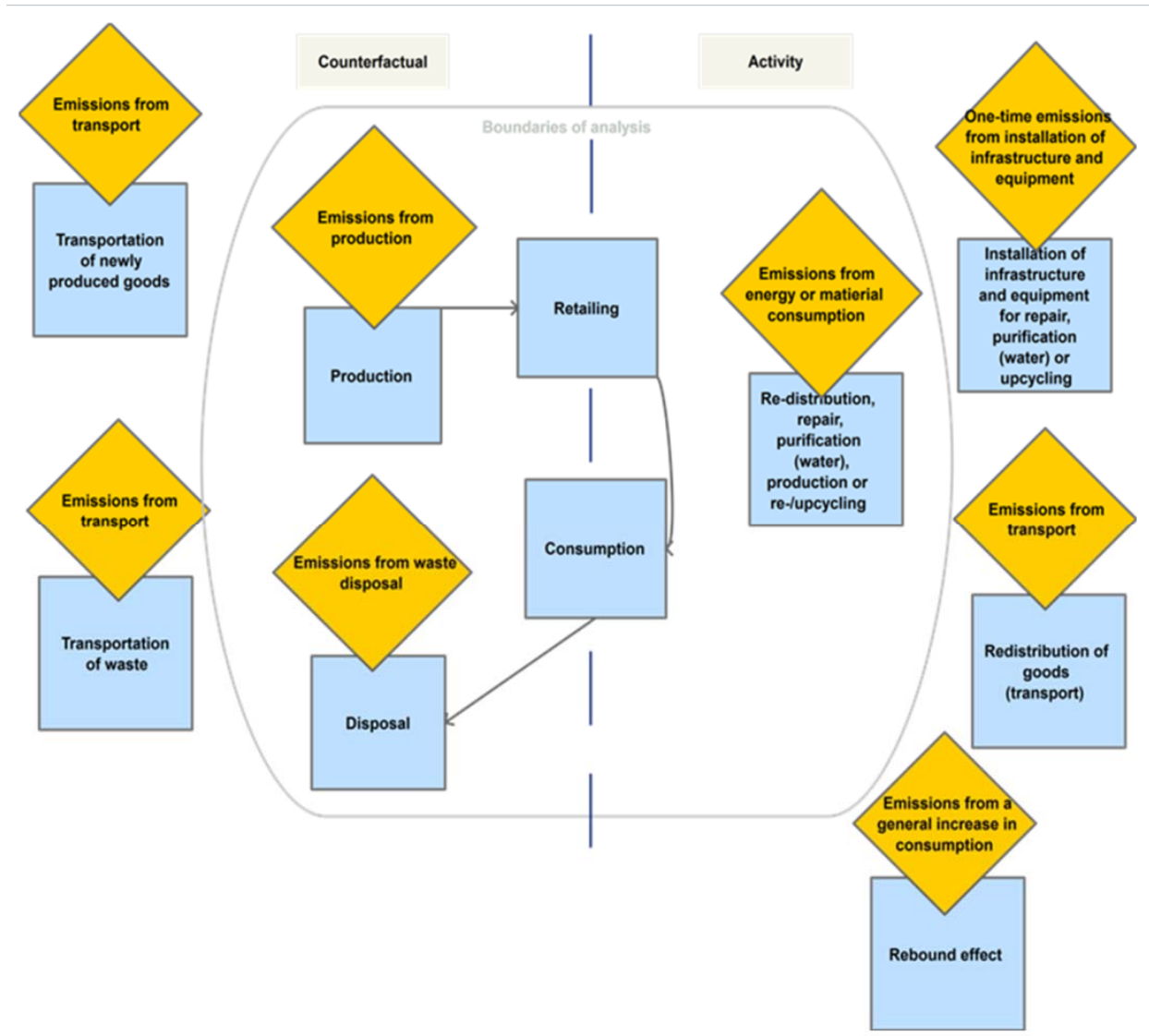

Fig. 1. Examples of GHG boundaries definition for counterfactuals and the CBIs'ELA.

\subsection{Towards an assessment across different activities: Defining unit-less indicators}

In regard of the issue introduced at point (3), there are several options through which is possible to operate a cross comparison of CBIs' EI that can be somehow significant. For example, a simple ratio as the one described in equation [1].

$$
\frac{E I_{C B I}}{E I_{c c}}
$$


Where: $\mathrm{EI}_{\mathrm{CBI}}$ is the $\mathrm{EI}$ associated to the $\mathrm{CBI}$, and $\mathrm{EI}_{\mathrm{CC}}$ is the $\mathrm{EI}$ associated to a conventional counterfactual.

However this can be valuable when comparing measures within the same domain/subdomain which will likely generate results in the same order of magnitude (at the same scale); however when the aim is the comparison across different domains, this although it is certainly the simplest - may not be the best option. In order to maintain an acceptable level of complexity and allowing cross-domain comparison the normalized difference approach is one option that can be proficiently applied to that purposes. It is borrowed from many applications in remote sensing analysis, and it is particularly suitable when the aim is to highlight the difference of two quantities (Lillesand R.W. 1994). It consists in a ratio that sees as numerator the difference of the EI associated to the $\mathrm{CBI}$ and the EI of the conventional counterfactual, whereas the denominator is the sum of the very same quantities.

$$
\frac{E I_{C C}-E I_{C B I}}{E I_{C C}+E I_{C B I}}
$$

This approach brings several advantages, in fact, besides being simple enough and not requiring more data than a simple ratio, it also perform better when aiming at crossdomain comparison. In fact it produces an index that ranges between -1 and 1 (Lillesand R.W. 1994); where values closer to +1 means that the level of EI associated with the activity(ies) of the CBI are smaller than the EI of the same activity(ies) if it was done by a standard counterfactual, thus implying that the CBI outperforms the counterfactual; conversely values closer to -1 indicate that the conventional counterfactual has actually lower EI than the CBI, thus indicating that in order to achieve an environmental benefit through this activity the CBI should improve its operational efficiency.

Table 2. List of activities and corresponding domains for which a specific EIA framework is provided

\begin{tabular}{|c|c|c|c|}
\hline Domain & Activities & Counterfactual & Equation \\
\hline Food & food production & $\begin{array}{lll}\begin{array}{l}\text { country } \\
\text { agriculture }\end{array} & \text { level } & \text { conventional }\end{array}$ & 3 \\
\hline Energy & $\begin{array}{l}\text { renewable energy production; } \\
\text { reduce energy demand }\end{array}$ & $\begin{array}{l}\text { country level energy production; } \\
\text { country level energy demand }\end{array}$ & 4 \\
\hline Transport & $\begin{array}{l}\text { food distribution; } \\
\text { goods/services provision; } \\
\text { people mobility }\end{array}$ & $\begin{array}{l}\text { fuel propelled means of transport } \\
\text { to provide an identical } \\
\text { service/good }\end{array}$ & 5 \\
\hline Waste & $\begin{array}{l}\text { recycling; } \\
\text { upcycling; } \\
\text { waste reduction } \\
\text { composting }\end{array}$ & country level waste treatment mix & 6 \\
\hline
\end{tabular}




\section{Materials and methods}

As previously anticipated in paragraph 2.2 the CBIs' inventory covers four main domains, and the most common activities carried out by CBIs (Tab. 2) are discussed below. In table 2 we listed some CBIs that focus on the direct production of a specific good while some other aim at delivering a set of services/benefits through alternative process which are generally considered to environmentally outperform standard procedures. Although these practices differ quite substantially, we conceptualise these as all proposing alternative-to-standard goods/services which are based on increased energy efficiency as shown in table 2. This is the case for example of urban agriculture, cohousing, or food distribution, which aim at providing a standard service with a smaller amount of inputs per unit of outputs. For such activities the choice of the counterfactual, as said, is fundamental; however, in literature we can find some feasible options. All the options explored below feature the normalized difference approach described with equation [2].

\subsection{Food Domain}

Generally speaking the CBIs operating in this domain mainly produce food themselves and/or focus on the distribution of food to members directly. To this domain belong all the CBIs operating an urban garden, managing a communal agricultural activity or running a SPG. Despite the several benefits that CBIs operating in this domain have on the socio-economic dimension (Mougeot 2005), the main GHG accountable output of CBIs involved in gardening is to produce food, usually organic, meant for auto-consumption or to serve local markets. Hence, the final good/service is to make members benefit from a certain food production. In order to investigate the associated EI, we inquire the CBI about the amount of resources used per unit of product, i.e. how much electricity (KWh) how much water (m3) how much and which kind of fertilizer (if any). These quantities can be then converted into amount GHG through adequate conversion factors that are country specific. In this case, the amount of emission related to agricultural production within each country per unit of product (http://www.faostats.org) is used as standard counterfactual (see eq. 3).

$$
\frac{G H G(A G)_{C C}-\left[G H G(E)_{C B I}+G H G(W)_{C B I}+G H G(F)_{C B I}\right]}{G H G(A G)_{C C}+\left[G H G(E)_{C B I}+G H G(W)_{C B I}+G H G(F)_{C B I}\right]}
$$

\section{Where:}

GHG(AG) $)_{\mathrm{CC}}$ is the amount of estimated $\mathrm{CO}_{2}$ equivalent emissions at the country level for crop cultivation,

$\mathrm{GHG}(\mathrm{E})_{\mathrm{CBI}}$ is the amount of $\mathrm{CO}_{2}$ equivalent of the $\mathrm{CBI}$ associated with energy use, $\mathrm{GHG}(\mathrm{W})_{\mathrm{CBI}}$ is the amount of $\mathrm{CO}_{2}$ equivalent of the $\mathrm{CBI}$ associated with water use, $\mathrm{GHG}(\mathrm{F})_{\mathrm{CBI}}$ is the amount of $\mathrm{CO}_{2}$ equivalent of the $\mathrm{CBI}$ associated with fertilization (if any), An example of how to estimate EI related to food production is given in eq. (3), whereas food distribution GHG accounting deserves a different framework. This activity in our inventory is carried out mainly by SPGs or similar CBIs. The main goal of an SPG is to distribute (often) local/organic/fair-trade food to its members possibly for a 
cheaper price. Hence, assessing the EI of distribution activity means to assess the GHG corresponding to the miles travelled by the food that the CBI delivers. Therefore, it consists in inquiring CBIs about distances, quantities, frequencies and means of transport corresponding to the food filling their basket (e.g. all food items or just the 10 most popular products), then calculate food miles, and consequently compare these with the food miles travelled by the same item to reach the country through standard market distribution, or alternatively to the proportion of GHG emissions imputable to transport in agriculture at the national level (Weber \& Matthews 2008) (which are generally around $20 \%$ of total agriculture GHG); .

This methodology can be proficiently applied to all CBIs active in the transport of goods, services or people through alternative and more sustainable ways (both means and/or infrastructure) than standard counterfactuals (see eq. 5), hence a more thorough depiction of its rationale is given in paragraph 3.3.

\subsection{Energy domain}

The CBIs of our inventory that operate in this domain can be grouped into two classes according to whether they focus mostly on sustainable energy production or on improving community energy efficiency.

To the first group belong the CBIs concerned with energy production through renewable sources (photovoltaic, biomass, wind etc.). Their activity is to bring together people in order to support the construction of a power (or heat) plant managed by the community itself. In this case, a viable option for EIA is to inquire CBIs about the amount of energy produced, estimate the level of GHG emissions associated with their energy production according to the type of resource utilized (DEFRA 2012), and using as a counterfactual the amount of GHG emissions imputable to the production of the same amount of energy if using the national grid system. However, communities that engage in energy production through renewable sources are very likely concerned with the EI of their energy consumption, thus adopting less demanding consumption behaviour. However, an EIA of this sort will result in neglecting to consider the GHG reduction deriving from more efficient consumption behaviour, focusing exclusively on the EI impact deriving from a more efficient production system.

Conversely, many CBIs such as co-housing initiatives, ecovillages, Transition towns etc., mainly focus on minimizing the demand of resources per capita (habitually electricity, water and fuels) through more sustainable consumption patterns. Thus, an EIA aiming at capturing this dimension of GHG reduction should inquire CBIs about the amount of resources (KWh of energy, $\mathrm{L}$ of fuels, $\mathrm{m}^{3}$ of water, etc.), the source of energy, and the number of participants, so to assess the total EI per capita within the initiative. In this case country level stats of the demand of the same set of resources per capita (and the associated GHG emissions) can serve as a counterfactual. However, an EI framed in this way will capture GHG reduction relative to sustainable consumption behaviour but may neglect to consider the energy spent for the production of the resource utilized.

In this regard, the EIA proposed in eq. (4) represents a valid option to calculate GHG reduction deriving from the potential combination (if the case) of renewable resource production and consumption behaviour at the same time. 


$$
\frac{\frac{G H G(E)_{C C}}{P O P_{C C}}-\frac{\sum_{i}^{n}\left(G H G(E)_{C B I}^{i} * A(E)_{C B I}^{i}\right)}{N P_{C B I}}}{\frac{G H G(E)_{C C}}{P O P_{C C}}+\frac{\sum_{i}^{n}\left(G H G(E)_{C B I}^{i} * A(E)_{C B I}^{i}\right)}{N P_{C B I}}}
$$

Where:

$\mathrm{GHG}(\mathrm{E})_{\mathrm{CC}}$ is the amount of $\mathrm{CO}_{2}$ equivalent linked to total energy consumption in the country $\mathrm{POP}_{\mathrm{CC}}$ is the population of the country

$\mathrm{GHG}(\mathrm{E})^{\mathrm{i}} \mathrm{CBI}$ is the $\mathrm{CO}_{2}$ emission conversion factor relative to the energy $i$ consumed by the $\mathrm{CBI}$ $\mathrm{A}(\mathrm{E})^{\mathrm{i}} \mathrm{CBI}$ is the amount of energy $i$ consumed by the CBI

$\mathrm{NP}_{\mathrm{CBI}}$ is the number of participants of the $\mathrm{CBI}$

Basically eq. [4] consists of comparing CBI's per capita resources consumption versus national per capita demand of the same resources taking into account also the efficiency of the production system.

\subsection{Transport (good/service/people mobility)}

This activity is operated by some CBIs whose aim is to deliver packages and goods minimizing the use of cars, trucks etc. and maximizing the use of low carbon mean of transport as bikes or human-powered trolleys (also for personal mobility). To calculate the beneficial EI reduction resulting from those activities the most reasonable option is represented by inquiring CBIs about the distance covered while moving/delivering goods/services/people, and using as a counterfactual the amount of GHG emissions released if the same distance was to be covered by fossil fuel propelled means of transport.

However, in order to investigate CBIs' EI in the transport domain the comparison of the GHG emission imputable to the CBI while covering a certain distance with the GHG emission corresponding the same distance if covered with "standard" fossil-fuelpropelled transports is considered a valid and reasonable proxy (see eq. 5).

$$
\frac{\left(D_{C B I} * E k m_{C C}\right)-\left(D_{C B I} * E k m_{C B I}\right)}{\left(D_{C B I} * E k m_{C C}\right)+\left(D_{C B I} * E k m_{C B I}\right)}
$$

\section{Where:}

$D_{C B I}$ is the distanced in $\mathrm{km}$ covered by the CBI

$E k m_{C C}$ is the energy in $\mathrm{KWh}$ necessary to cover $1 \mathrm{Km}$ or the estimate of the $\mathrm{CO}_{2}$ equivalent emitted to cover $1 \mathrm{~km}$ by the counterfactual (i.e. with fossil fuels propelled mean of transport.

$E k m_{C B I}$ is the energy in $\mathrm{KWh}$ necessary to cover $1 \mathrm{Km}$ or the estimate of the $\mathrm{CO}_{2}$ equivalent emitted to cover $1 \mathrm{~km}$ by the CBI (usually with human powered mean of transport).

$E_{C B I}$ is the energy in $\mathrm{KWh}$ (or the estimate of the corresponding $\mathrm{CO}_{2}$ equivalent emitted) spent by the CBI to operate its activities.

\subsection{Waste treatment (reusing/recycling/upcycling and composting)}

The activities belonging to the waste domain are often the object of a harsh debate because of the variables and limiting factors that influence an intelligible EI assessment. However, we do not have to investigate which waste management option is 
more environmental friendly, rather to focus on assessing the environmental benefit (if any) of the activities dealing with waste in our inventory. In this regard, whether the CBI treats exclusively organic waste, or hard-dry materials or a combination of both makes a relevant difference for the choice of the counterfactual, thus potentially affecting whether the CBI's has a positive or negative outlook.

For instance, in case the activity treats also organic waste also composting needs to be considered, either as a CIB activity or as a counterfactual. In this regard some scholars consider composting a very efficient carbon sink, although some other advocate that organic fermentation (through composting) generates a plethora of GHGs (e.g. $\mathrm{CH}_{4}$, $\mathrm{N}_{2} \mathrm{O}$ etc.) whose effects are even worse than $\mathrm{CO}_{2}$, thus balancing off the positive EI represented by locking up carbon in topsoils. Besides, although landfilling harms ecosystems in several ways (i.e. it is responsible for the contamination of water and land), it is also supposed to be a very efficient carbon sink which store carbon for a longer time than composting. Studies show that pyrolisation and incineration are better waste management types since they use waste as fuel to produce energy, and the net balance of $\mathrm{CO}_{2}$ emissions support the claim that it is less costly in term of $\mathrm{CO}_{2}$ emissions than conventional energy from fossil fuel(Pachauri \& Reisinger 2007).

However, a more detailed discussion of this matter is given in paragraph 5; in fact, regardless of what literature says about advantages and disadvantages of different waste management type, our aim is to develop an EIA that can achieve a good degree of similarity with the methods proposed to calculate EI in the other domains. Thus, the rationale used for waste simply answers the question "what would have happened to the CBI's waste if not regenerated/treated by the CBI?". It is reasonable to think that it would be treated according to the mix of management types used within the context region; hence, country level data about waste treatment mix can be used as a proxy when finer scale data are not available.

Besides, waste recycling/upcycling activities require a certain amount of energy to transform waste into usable and valuable product. Hence, also the $\mathrm{CO}_{2}$ eq. imputable to that energy demand shall be included in the calculation (see eq. 6).

$$
\left.\frac{\sum_{i}^{n}\left[W R_{C B I} * P M_{C B I}^{i} * G H G(C)^{i}\right]-\left\{\sum_{i}^{n}\left[W R_{C B I} * P M_{C B I}^{i} * G H G(C)^{i}\right]+G H G(E)_{C B I}\right\}}{\sum_{i}^{n}\left[W R_{C B I} * P M_{C B I}^{i} * G H G(C)^{i}\right]+\left\{\sum_{i}^{n}\left[W R_{C B I} * P M_{C B I}^{i} * G H G(C)^{i}\right]+G H G(E)_{C B I}\right.}\right\}
$$

Where:

$W \mathrm{R}_{C B I}$ is the amount of waste (in mass unit) "regenerated" by the CBI

$P M^{i}{ }_{C C}$ is the proportion of waste that is processed by the counterfactual with the $i^{\text {th }}$ management type, given that in the country/region/community waste are treated in $n$ possible ways, $P M^{i}{ }_{C B I}$ is the proportion of waste that is processed by the CBI with the $i^{\text {th }}$ management type, given that in the country/region/community waste are treated in $n$ possible ways, $G H G(C)$ is the conversion factor to $\mathrm{CO}_{2}$ eq. emissions per mass unit for the management type $i$. $G H G(E)_{C B I}$ is the amount of GHG emission produced by the energy demand of the CBI. 


\section{Results}

We applied the methods described in paragraph 3 to assess the EI of a few case studies. For each case study, we will highlight key aspects that we believe interesting to present and discuss.

\subsection{Community bike repair shop: waste domain}

The first type of CBI is a 'community bike repair shop'. The TESS project mapped several of them across Europe. These CBIs search garbage for trashed bikes spare parts and broken items that can be repaired/reused/recycled to make usable bikes or used as replacements. They repair broken bikes and give out spare parts for free in order to allow anyone in the community the possibility to use a bike (the results here presented refer to one community bike repair shop particularly relevant and active in the centre of Rome). In regards of calculating EI, the main direct impact of these CBIs deals with the amount of waste they recycle, whereas indirectly they create a movement of people (or group of interest) that use bikes as the main mean of transport in every-day life. In regards of the impact deriving from the recycling activity, the CBI declared of being capable of regenerating 7 working bikes out of 10 trashed bikes. Given that they collect about $1500 \mathrm{~kg}$ of solid waste per year under the forms of broken bikes or parts, the CBI recycles directly $1,050 \mathrm{~kg}$ ( $70 \%$ of total) of solid waste per year while the remaining $450 \mathrm{~kg}$ are sent to the municipal waste facility and treated accordingly. Hence, as a standard counterfactual it is reasonable to take average municipal waste emissions per management type in Europe and apply these values to the mix of municipal waste treatment in Italy. According to the European Environment Agency(European Environment Agency 2010) in Europe the treatment of municipal waste in 2010 was roughly 210 million tonnes (92 $\mathrm{Ml} \mathrm{T}$ landfilling, $57 \mathrm{Ml} \mathrm{T}$ incineration and $62 \mathrm{Ml} \mathrm{T}$ recycling, see tab. 3). Net GHG emissions per $\mathrm{kg}$ of waste resulted to be $0.79 \mathrm{CO}_{2}$ eq. for landfilling, $0.02 \mathrm{CO}_{2}$ eq. for incineration, and $-0.75 \mathrm{CO}_{2}$ eq. for recycling(European Environment Agency 2010). Thus, according to eq. (6) we can compare the EI corresponding to the amount of waste processed by the CBI in a year if it was treated with the disposal mix of Italian municipal waste $(56.53 \%$ landfilled, $20.48 \%$ incinerated, $22.99 \%$ recycled), with the EI of the waste management type associated with CBI's activity (16.96\% landfilled, $6.14 \%$ incinerated, $76.9 \%$ recycled). 
Table 3. Coefficients used to assess EI for the CBI operating in the waste domain and its counterfactual (data refer to 2010 and are available through the Eurostat online database, data in italics are personal elaboration).

\begin{tabular}{|c|c|c|c|c|c|}
\hline Item & Landfilling & Incineration & Recycling & Total & Units \\
\hline EU direct emission & 76.31 & 21.58 & 27.02 & & (Million tonnes) \\
\hline EU avoided emission & -3.65 & -20.69 & -73.47 & & (Million tonnes) \\
\hline EU total municipal waste & 92.00 & 57.00 & 62.00 & & (Million tonnes) \\
\hline EU net GHG emission & 0.79 & 0.02 & -0.75 & & $\left(\mathrm{CO}_{2}\right.$ eq. $\left./ \mathrm{kg}\right)$ \\
\hline Waste management type & & & & & \\
\hline Italy & 56.53 & 20.48 & 22.99 & & $(\%)$ \\
\hline $\begin{array}{l}\text { Waste management type } \\
\text { CBI }(\%)\end{array}$ & 16.96 & 6.14 & 76.90 & & $(\%)$ \\
\hline $\begin{array}{l}\text { GHG of the counterfactual to } \\
\text { treat CBI yearly waste }\end{array}$ & 1073 & 19738 & -460 & 20351 & (kg CO $\mathrm{CO}_{2}$ eq.) \\
\hline $\begin{array}{l}\text { GHG of the CBI to treat } C B I \\
\text { yearly waste }\end{array}$ & 322 & 5921 & -1539 & 4704 & $\left(\mathrm{~kg} \mathrm{CO}_{2}\right.$ eq. $)$ \\
\hline
\end{tabular}

In total the $\mathrm{CBI}$ is responsible for $\sim 5$ tonnes of $\mathrm{CO}_{2}$ eq. per year, while for the same amount of waste the standard counterfactual emits $\sim 20$ tonnes of $\mathrm{CO}_{2}$ eq. GHG 4 times more. The resulting EIA calculated according to eq. (6) is 0.62 , thus showing that the CBI outperforms the standard counterfactual.

\subsection{Bicycle use promoting initiative: Transport domain}

The second domain for which case study results are presented is the transport domain. CBIs operating in this domain do several different activities; however goals are pretty much similar and consist in promoting bike usage through the implementation of suitable condition for bike friendly environment. In Rome, one CBI was able to monitor that its members (roughly 1,136) covered more than 160 thousands kilometres on bikes during May 2014 (exactly 167,330 Km). In order to provide EIA according to eq. [5] the counterfactual must be designed in a way that elicits the GHG emission corresponding to that aggregate distance if it was to be covered with a "standard" car. We opted for a conservative estimate, so we defined as "standard" a vehicle respecting the most recent regulation imposed by the European Union to all new passenger cars by 2015 (130 g of $\mathrm{CO}_{2}$ per $\mathrm{Km}$ ) (Cuenot 2009; Fontaras \& Samaras 2010). Thus, the $\mathrm{CO}_{2}$ eq. that would have been consumed by a counterfactual (or that has been saved by the $\mathrm{CBI}$ ) adds up to 21.75 tonnes of $\mathrm{CO}_{2}$ eq. under the assumption that every bike kilometre would have been covered by car (i.e. $130(\mathrm{~g} / \mathrm{Km}) * 167,330(\mathrm{Km})=21.75 \mathrm{~T})$. Besides, to fulfil the variables required in eq. (5) we need to know also the amount of energy spent by the CBI in conducting its activity. However, the structure of this CBI is extremely liquid hence having a clear picture of the amount of time and energy spent to manage its activity is somewhat elusive; yet, we can speculate that all participants had connected a standard pc for a couple of hours per day (rough estimate coming from interviews) to promote/manage the activity during that month (average consumption of 120 watts, Intel report from Trent University web page). Consequently, given that the GHG emission factor for national electricity in Italy is $\sim 406$ grams of $\mathrm{CO}_{2}$ per $\mathrm{KWh}$ we 
estimated that CBI's GHG emission would be $\sim 3$ tonnes of $\mathrm{CO}_{2}$ eq. (1050 PCs, running $2 \mathrm{~h}$ per day for 30 days with an average consumption of 120 watts). Thus, EIA for this CBI according to eq. [6] is 0.75 suggesting that the activity promoted by such CBIs represent a relevant improvement in reducing EI if compared to a standard counterfactual.

\subsection{Solar energy initiative}

As stated before, CBIs active in the energy domain are involved in two main activities. First, they engage in the production of renewable energy and second they work on increasing energy efficiency. In our repository we had one CBI from Germany (constituted by a group of university students and other interested members) that installed a photovoltaic power plant on the roof top of university buildings. In 2013 they produced roughly $30 \mathrm{MWh}$ of electricity, feeding it into the power grid. Emissions from the electricity production with PV power plants have been analysed in a critical metastudy taking a full life cycle approach proposed by Nugget and Sovacool in 2014(Nugent \& Sovacool 2014). They find average values of roughly $80 \mathrm{~g} \mathrm{CO}_{2} / \mathrm{kWh}$ produced. Thus the energy production is equivalent to emissions of 30 (MWh) * $1000(\mathrm{KWh} / \mathrm{MWh}) *$ $0.08(\mathrm{Kg} \mathrm{CO} 2 / \mathrm{KWh})=2,4\left(\mathrm{~T} \mathrm{CO}_{2}\right)$. As counterfactual, we take electricity supplied from the national grid. The emissions factors for electricity in Germany is $0.477\left(\mathrm{Kg} \mathrm{CO}_{2}\right.$ / KWh) (DEFRA 2012; IEA 2010), this results in emissions for the counterfactual of 30 $(\mathrm{MWh}) * 1000(\mathrm{KWh} / \mathrm{MWh}) * 0.477(\mathrm{Kg} \mathrm{CO} 2 / \mathrm{KWh})=14,3\left(\mathrm{~T} \mathrm{CO}_{2}\right)$. Hence, the resulting EIAs in $\sim 0.71$, thus suggesting that the activity carried on by the $\mathrm{CBI}$ is responsible for a relevant reduction of GHG emissions (per unit of product).

\subsection{General remarks}

One of the important contributions of the results presented above relies in the fact that, besides presenting a methodology for an EIs' evaluation of CBIs that is crossactivity, its preliminary findings also suggest that in all cases (investigated here) the alternative methods through which CBIs carry out their activities lead to outperform the respective standard counterfactual. The three examples described in sections 4.1, 4.2 and 4.3 elicit an indicator of EIA of CBIs spanning between circa 0.6 and 0.75 , therefore suggesting that the potential contribution in reducing GHG from CBIs per unit of output is substantial. This is particularly relevant especially because the 2 out of 3 of the examples given pertain to activity sectors in Europe that are responsible for a large share of GHG (Fig. 2); in fact the transport domain contributes for a little less than $20 \%$ of total European GHG, the energy production domain contributes for something slightly above $30 \%$, while the waste domain is responsible for only $\sim 3 \%$. According to our analytic framework, the 3 examples given above may be associated with a percentage reduction of GHG per unit of output (Tab. 4) ranging from $\sim 75 \%$ to $\sim 85 \%$; these are remarkable results, that given current European GHG level, efforts in filling the gap from current GHG with expected GHG according to the Agenda 2020, suggest that CBIs potential contribution to mitigating climate change target is no longer negligible. On the other hand, is true that the examples above are very small case studies that can be considered the "standard" among CBIs' practices. Therefore the potential aggregate benefits deriving from upscaling their activities at a larger societal scale is an issue that needs to 
be addressed thoroughly looking also at the many caveats that this type of investigation may convey. This and other elements that can contribute fruitfully to a constructive discussion on the subject are further presented in section 5 .

Table 4. Results of the environmental evaluation framework proposed for the 3 CBIs used as case studies eliciting EIA and fraction of GHG reduction.

\begin{tabular}{|c|c|c|c|}
\hline Domain & CBI's activity & EIA & GHG reduction $(\%)$ \\
\hline & $\begin{array}{l}\text { Community bike } \\
\text { repair shop }\end{array}$ & 0.62 & $75 \%$ \\
\hline Transport & $\begin{array}{l}\text { Bicycle use } \\
\text { promoting } \\
\text { initiative }\end{array}$ & 0.71 & $83 \%$ \\
\hline $\begin{array}{c}1 \\
\text { Energy }\end{array}$ & $\begin{array}{l}\text { Solar Energy } \\
\text { Initiative }\end{array}$ & 0.75 & $85 \%$ \\
\hline
\end{tabular}

\section{Discussions}

The EIA framework proposed raises some interrogatives dealing with uncertainty under different perspectives. On the one hand general reflections on the validity of the tool proposed to address GHG reduction at a global/region (macro scale) can be made; while on the other hand some skepticism may arise on the legitimacy of the assumptions taken to build appropriate counterfactuals (micro scale).

\subsection{Macro scale issues}

Figure 2a (data elaborated by the EEA in 2010. European Environment Agency 2010) shows that environmental goals still sit quite far for all European countries, even for the northern European virtuous Republics (Sweden and Denmark above all). The composition of GHG by sector in Europe elicited in figure 2b (European Environment Agency 2010), makes clearly visible how some domains have a greater potential than others, hence, direct EI of some CBIs may result negligible no matter how efficient the adopted practice is. For example, the waste domain accounts for less than $3 \%$ of total GHG in Europe (Fig. 2b), hence even if transition to a zero waste practice would be largely adopted repercussion on total GHG cannot yield to relevant results. Conversely also small percentage reduction of GHG in the transport or energy domain may elicit a great step forward in achieving environmental goals. In view of the strong lock-in of the transport system in terms of infrastructure and institutions, experimentation and development of niches is especially important. With no surprise in fact the potential of 
CBIs for niche development has been stressed multiple times by several scholars (Seyfang and Smith 2007).
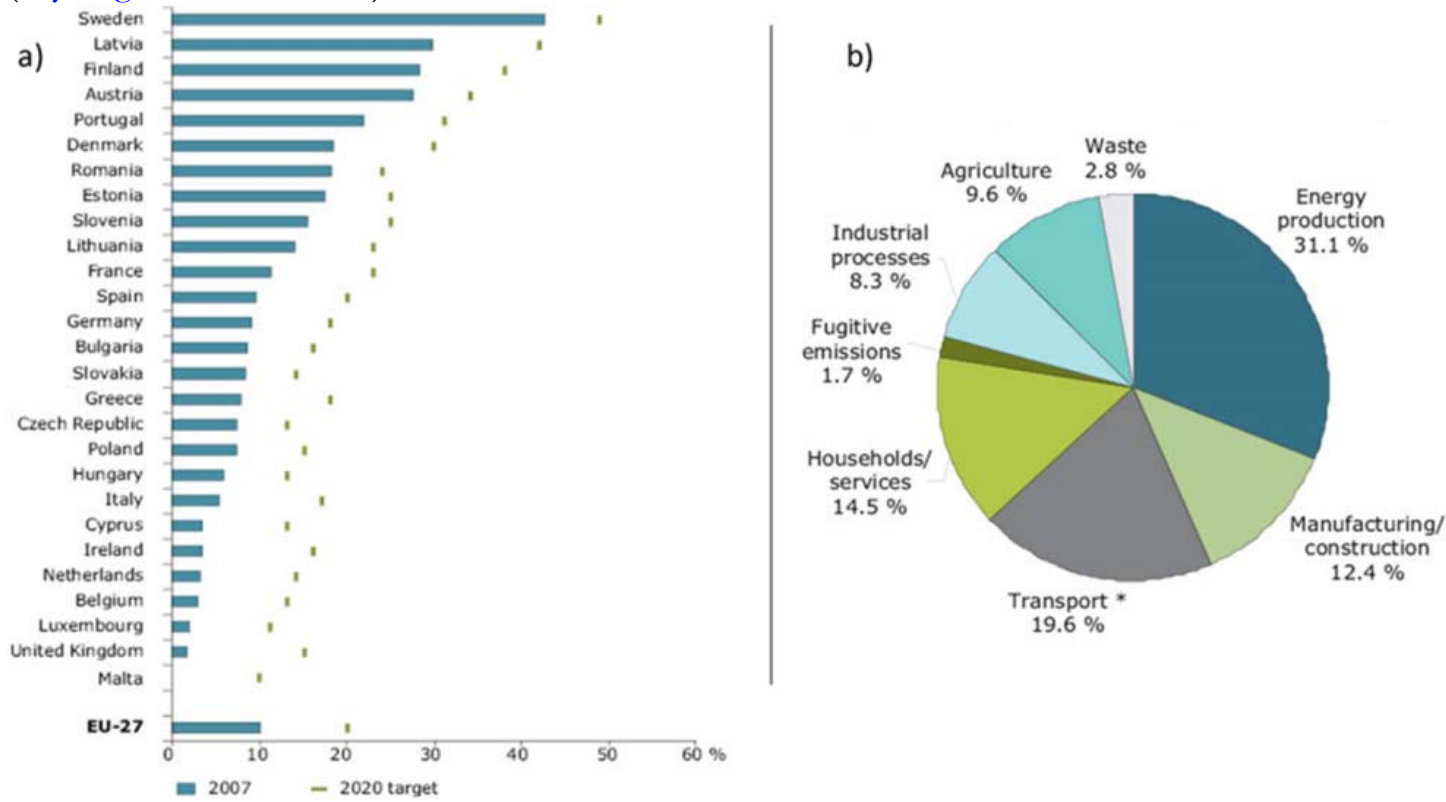

Fig. 2. On the left (a) "Share of renewable energy in final energy consumption in EU-27 in 2007 compared to 2020 targets". On the right (b) "Total greenhouse gas emissions by sector in EU-27 in 2008". Source: European Environment Agency - State and Outlook 2010.

Furthermore, although according to our calculation CBIs' activities are in general more efficient than the corresponding standard counterfactual (less $\mathrm{CO}_{2}$ eq. emitted per unit of product/service); the magnitude of their impact may not be relevant given the limited size of the $\mathrm{CBI}$ (total $\mathrm{CO}_{2}$ eq. the $\mathrm{CBI}$ saves compared to total $\mathrm{CO}_{2}$ eq. the regional counterfactual is responsible for). To this end, another limitation arises: in fact to assess the total impact of a specific activity in one domain we should not account solely for one single CBI but on all CBIs operating in the same domain in the same area. Thus, more structured analysis focusing on specific case study areas are needed in this sense. However this is not the object of this study; in here we aim at proposing a methodologic framework for EIA of the activities of grass roots initiatives, whereas in depth regional investigation will follow.

\subsection{Micro-scale issues}

In regard of micro scale issues of concern, arguments are presented example by example.

- $\quad$ Bike repair shop (waste): The example presented within the waste domain does not distinguish between hard solid materials and organic "humid" waste; hence an EIA for organic waste composting is not described. However, TESS mapped several CBIs promoting the activity of collecting and processing organic waste in order to transform it 
into valuable minerals-rich soil. Although this practice is habitually associated with food oriented initiatives, the method presented in paragraph 3.4 may be suitable also for such upcycling practice (it can be considered as the $i$ th among $n$ waste management types). This practice is a natural mean for stocking large amount of carbon; however we have to note though, that whether composting's EI outperforms a standard counterfactual's EI is still a debated topic(Pachauri \& Reisinger 2007; DEFRA 2012). In fact, it is supposed to potentially reduce GHG emissions only in the long run, whereas in the short/medium run both incineration and landfilling (when coupled with gas capturing) show lower GHG emissions(Pachauri \& Reisinger 2007). Studies show that time plays a crucial role in establishing which of the waste treatment method is less harmful for the environment, but these short and long run scenarios all agree in showing that incineration is always better than the other methods (recycling is not considered), also if the additional $\mathrm{CO}_{2}$ eq. net reduction imputable to energy production is not considered(Pachauri \& Reisinger 2007; DEFRA 2012; Mohareb et al. 2011). However, as a counterargument is habituallysaid that burning matters would make sense only if we could dispose of more than one planet, thus, speculating that burning and transforming matter into energy would be reasonable if our planet could provide an infinite amount of resources. In conclusion literature does not offer a clear picture of which option is better, hence finding a reasonable counterfactual is a critical issue that deserve to be investigated through ad hoc further research. On another note, the EIA for the community bike repair shop operating in the waste domain tells us that the CBI is substantially more efficient than the counterfactual, however in a year the CBI is able to treat an amount of waste only equivalent to what is trashed by 3 people ( $500 \mathrm{Kg} /$ per cap., (Eurostat 2010$)$ ), thus its contribution to lower GHG is minimal (this relates to macro scale issue in paragraph 5.1). Nonetheless, we observed that such CBIs often became recognised places of attraction catalysing large interest groups. Habitually these people make an intense use of the bike as the main mean of transport. The CBI presented in the example have been able through the last few years to attract a core of about 1000 people whose aim is to replace at most fossil fuel propelled personal mobility with cycling. The indirect EI that this phenomenon represents may be responsible for a substantial reduction of GHG in the transport domain. In fact, EI reduction could be of a magnitude comparable to what has been described for the CBI active in the transport domain (paragraph 4.2). Thus, since indirect impact may be extremely relevant in lowering GHG at the aggregate scale, a structured analysis on this subject should not be neglected.

- Transport: The method proposed for EI assessment of CBI in the transport domain may also raise some scepticism. In fact, the assessment of transport GHG framed as the $\mathrm{CO}_{2}$ equivalent corresponding to the energy needed to cover a certain distance $(\mathrm{KWh} / \mathrm{Km})$ conveys some caveats. In the case that the CBI uses sustainable transport to deliver goods or services one could speculate that using a car/truck allows to carry a larger volume of goods per trip, hence reducing the total number of $\mathrm{Km}$ travelled by the counterfactual; conversely some cohorts may advocate that bike transport can access roads usually unavailable for conventional car/trucks (i.e. restricted traffic areas, narrow lanes or bridges etc.) thus allowing the CBI to cover shorter distances. Furthermore, the variables taken to calculate CBI's energy demand are based 
on hypothesis that - although being reasonable - still need to be validated. Moreover, once data collection will be completed this part of the study will definitely benefit from more in depth investigation, and a sensitivity analysis will be conducted.

- $\quad$ Energy: Regarding the example given in paragraph 4.3 about photovoltaic energy production on campus, some concerns may arise due to the appropriateness of the activity boundaries chosen, given the main vocation of the CBI. In fact, members of the CBI made very clear during interviews that energy production is neither their main goal nor their principal activity; conversely they mainly aim at rising sustainability awareness on/off campus and at making their university a more environmental friendly place. To this end, on top of installing solar panels on campus they provide/organize a number of seminars, lectures and events to raise awareness about environmental issues. Unfortunately, no data is available on the resulting behavioural change the CBI may have influenced. Therefore, we cannot determine the EI related to their main activity.

We found this issue as very common among the CBIs populating our inventory in fact most of them do or participate to dissemination/educational activities. The degree of involvement in such activities - and analogously the effect produced - is different for all of them, which somehow is a problem that encompasses all the three caveats presented in paragraph 2. Probably this is the principal source of uncertainty of the framework proposed and one of the obstacles that further research shall try to exhaustively address. Nevertheless, it translates in the fact that if on the one hand we are capable of elaborating reasonably robust and valid methodology to provide EIA for most of the practices resulting in a clear GHG accountable output, on the other hand there is a lack of sound and justified hypothesis about dissemination and educational activities onto which build a similar assessment framework (Uwasu et al. 2009). We believe that this gap needs to be closed in order to provide fully informed support for sustainable aware policy making (Meadowcroft 2011; Meadowcroft 2009; O'Riordan 2004); therefore, the synergic effort of environmental humanities and natural sciences is fundamental in this regard (Loorbach 2010). The theoretical and methodological framework developed with this study aims at being a step towards in closing the gap in the literature about CBIs. This will give CBI's a stronger voice by providing them the opportunity to support their contribution to a transition in Europe with comparable numbers. Moreover, it will allow uncovering areas with large potentials.

\section{Conclusions}

The rationale described and the examples given aim at defining some guidelines for EIA that can be useful at multiple level. First, as shown, this framework finds suitable application in case studies and assessment reports for researchers and scholars investigating the theoretical background of CBIs' EI. Second, applications in societal planning and design would particularly benefit because it represent a valid tool onto which CBIs' activities are rated. Thus it could be used to evaluate the potential effect and consequences of scaling-up scenarios. Third, it is informative of the comparative performance of different activities a CBI may conduct, hence representing a valid tool to 
identify which activity outperform the others in fostering sustainability, thus suggesting which one should be improved or prioritized.

In conclusion, we acknowledge that CBIs' activities encompass very diverse situations and consequently are strongly influenced by loco-regional conditions; hence any attempt to perform a cross regional comparison must ground on generalizations that avoid oversimplifications and distortions. However, we believe that the theoretical framework proposed is suitable to produce meaningful results. Furthermore, despite the many uncertainties discussed in our study it conveys three major implications that improve the discourse about CBIs' environmental impacts. First, our results suggest that policy makers and scholars should not solely consider direct impact of CBIs' activities, because indirect impact may reveal to have a greater potential in fostering sustainable transition, and thus should not be neglected. Second, the choice of the counterfactual is a task that must be carried out carefully and communicated transparently since its definition may greatly influence EIA. Third, CBIs operating in certain domains such transport and energy have theoretically a greater potential in producing sensible results; in fact a cut of a certain percentage of GHG in the waste domain won't produce relevant results in absolute terms as a cut of the identical percentage in the transport or energy domains.

\section{Acknowledgments}

We would like to thank all our colleagues that were involved in the TESS activities for the inspiring discussion and reflections, in particular Jürgen P. Kropp, Anne Holsten, and David Landholm (Potsdam Institute for Climate Impact Research). Moreover, we would like to mention and thank Charlie Henderson and Iga Gozdowska (Climate Futures), and Cary Hendrickson (University of Roma La Sapienza) for their valuable contribution in drafting a previous extended abstract of this paper that was presented at the European Geophysical Union general assembly in 2015. Furthermore, we want to acknowledge that the research leading to these results has received funding from the European Community's Seventh Framework Programme under Grant Agreement No. 603705 (Project TESS). This manuscript reflects the authors' views. The European Commission is not liable for any use that may be made of the information contained therein.

\section{References}

Brown, J.R., 2004. Comparative Analysis of Energy Consumption Trends in Cohousing and Alternate Housing Arrangements.

Cembalo, L., Migliore, G. \& Schifani, G., 2013. Sustainability and New Models of Consumption: The Solidarity Purchasing Groups in Sicily. Journal of Agricultural and Environmental Ethics, 26(1), pp.281-303.

Chavez, A. \& Ramaswami, A., 2011. Progress toward low carbon cities: approaches for transboundary GHG emissions' footprinting. Carbon Management, 2(4), pp.471-482.

Church, C. \& Elster, J., 2002. Thinking locally, acting nationally Lessons for national policy from work on local sustainability, York. Available at: http://www.jrf.org.uk/sites/files/jrf/1842631381.pdf. 
Conrad, C.C. \& Hilchey, K.G., 2011. A review of citizen science and community-based environmental monitoring: Issues and opportunities. Environmental Monitoring and Assessment, 176(1-4), pp.273-291.

Cuenot, F., 2009. CO2 emissions from new cars and vehicle weight in Europe; How the EU regulation could have been avoided and how to reach it? Energy Policy, 37(10), pp.3832-3842.

Daviet, F. \& Ranganathan, J., 2005. The Greenhouse Gas Protocol: The GHG Protocol for Project Accounting. The Project Protocol. Available at: http://pdf.wri.org/ghg_project_accounting.pdf.

DEFRA, 2012. Guidelines to Defra/DECC GHG Conversion Factors for Company Reporting. Department of Energy and Climate Change, pp.1-54.

European Environment Agency, 2010. Synthesis 46 The European environment | State and outlook 2010. European Environment, pp.46-67. Available at: http://library.iyte.edu.tr/dosya/kitap/soer2010.pdf.

European Environmental Agency, 2013. Annual European Union greenhous gas inventory 1990-2011 and inventory report 2013. EEA Technical report, 8, p.22.

Eurostat, 2010. Europe in Figures: Eurostat yearbook 2010, Available at: http://www.lavoisier.fr/notice/frEZORRO2TLXTALO.html.

Finnveden, G. \& Moberg, A., 2005. Environmental systems analysis tools - an overview. Journal of Cleaner Production, 13(12), pp.1165-1173. Available at: http://dx.doi.org/10.1016/j.jclepro.2004.06.004.

Fontaras, G. \& Samaras, Z., 2010. On the way to $130 \mathrm{~g} \mathrm{CO} 2 / \mathrm{km}$-Estimating the future characteristics of the average European passenger car. Energy Policy, 38(4), pp.1826-1833.

Forrest, N., 2011. Discovering Pathways to Sustainability: Small Communities in Transition. Dissertation, (August).

Galan, M.B., Peschard, D. \& Boizard, H., 2007. ISO 14001 at the farm level: Analysis of five methods for evaluating the environmental impact of agricultural practices. Journal of Environmental Management, 82(3), pp.341-352.

Galli, A. et al., 2012. Integrating Ecological, Carbon and Water footprint into a "footprint Family" of indicators: Definition and role in tracking human pressure on the planet. Ecological Indicators, 16, pp.100-112.

Gibson, R.B., 2006. Sustainability assessment: basic components of a practical approach. Impact Assessment and Project Appraisal, 24(3), pp.170-182.

IEA, 2010. Key World Energy Statistics 2010, Available at: http://www.oecd-ilibrary.org/energy/key-worldenergy-statistics-2009_9789264039537-en.

Kennedy, S. \& Sgouridis, S., 2011. Rigorous classification and carbon accounting principles for low and Zero Carbon Cities. Energy Policy, 39(9), pp.5259-5268.

Li, L.W. et al., 2013. Transitioning to Community-owned Renewable Energy: Lessons from Germany. Procedia Environmental Sciences, 17, pp.719-728. Available at: http://linkinghub.elsevier.com/retrieve/pii/S1878029613000911 \nhttp://www.sciencedirect.co $\mathrm{m} /$ science/article/pii/S1878029613000911.

Lillesand R.W., T.M. and K., 1994. Remote sensing and image interpretation -- 3rd ed. John Wiley and Sons, Inc., New York, pp.524-647.

Loorbach, D., 2010. Transition Management for Sustainable Development: A Prescriptive, ComplexityBased Governance Framework. Governance: An International Journal of Policy, Administration, and Institutions, 23(1), pp.161-183. Available at: http://doi.wiley.com/10.1111/j.14680491.2009.01471.x.

Manuilova, A., Suebsiri, J. \& Wilson, M., 2009. Should Life Cycle Assessment be part of the Environmental Impact Assessment? Case study: EIA of CO2 Capture and Storage in Canada. In Energy Procedia. pp. 4511-4518.

Marcus, A. \& Wagner, F., Realizing Utopia: Ecovillage Endeavors and Academic Approaches RCC. A. Marcus \& F. Wagner, eds., Munich: Rachel Carson Center for Environment and Society. Available at: http://www.environmentandsociety.org/perspectives/2012/8/realizing-utopia-ecovillageendeavors-and-academic-approaches.

Meadowcroft, J., 2011. Engaging with the politics of sustainability transitions. Environmental Innovation and Societal Transitions, 1(1), pp.70-75.

Meadowcroft, J., 2009. What about the politics? Sustainable development, transition management, and longterm energy transitions. Policy Sciences, 42(4), pp.323-340. 
Mohareb, E.A., Maclean, H.L. \& Kennedy, C.A., 2011. Greenhouse Gas Emissions from Waste Management - Assessment of Quantification Methods. Journal of the Air Waste Management Association, 61(5), pp.480-493. Available at: http://www.tandfonline.com/doi/abs/10.3155/1047-3289.61.5.480.

Möllersten, K. \& Grönkvist, S., 2007. All CO2 is equal in the atmosphere-A comment on CDM GHG accounting standards for methane recovery and oxidation projects. Energy Policy, 35(7), pp.36753680.

Mougeot, L.J.A., 2005. Agropolis: The Social, Political and Environmental Dimensions of Urban Agriculture, Available at: http://www.idrc.ca/openebooks/186-8/.

Network, G., 2001. Community Sustainability Assessment (CSA), Available at: http://scholar.google.com/scholar?hl=en\&btnG=Search\&q=intitle:Community+Sustainability+ Assessment $+(\mathrm{CSA}+) \# 1$.

Nugent, D. \& Sovacool, B.K., 2014. Assessing the lifecycle greenhouse gas emissions from solar PV and wind energy: A critical meta-survey. Energy Policy, 65, pp.229-244.

O'Riordan, T., 2004. Environmental science, sustainability and politics. Transactions of the Institute of British Geographers, 29(2), pp.234-247.

Ostrom, E., 1990. Governing the Commons: the evolutions of institutions for collective action, Available at: http://markdaku.com/poliscinotes/?p=173\nhttp://fr.wikipedia.org/wiki/Trag?die_des_biens_c ommuns.

Pachauri, R.K. \& Reisinger, A., 2007. IPCC fourth assessment report. IPCC Fourth Assessment Report. Available http://www.construible.es/construible \biblioteca \presentacion_informe_ipcc.pdf $\backslash$ npapers2://p ublication/uuid/DD3ABB67-E411-4C0F-A29C-DA693B95B789.

Peigné, J. \& Girardin, P., 2004. Environmental impacts of farm-scale composting practices. Water, Air, and Soil Pollution, 153(1-4), pp.45-68.

Roseland, M., 2000. Sustainable community development: Integrating environmental, economic, and social objectives. Progress in Planning, 54(2), pp.73-132.

Saer, A. et al., 2013. Life cycle assessment of a food waste composting system: Environmental impact hotspots. Journal of Cleaner Production, 52, pp.234-244.

Seyfang, G. \& Haxeltine, A., 2012. Growing grassroots innovations: Exploring the role of community-based initiatives in governing sustainable energy transitions. Environment and Planning C: Government and Policy, 30(3), pp.381-400.

Sultana, F., 2009. Community and participation in water resources management: Gendering and naturing development debates from Bangladesh. Transactions of the Institute of British Geographers, 34(3), pp.346-363.

Takeuchi, K., 2010. Rebuilding the relationship between people and nature: The Satoyama Initiative. Ecological Research, 25(5), pp.891-897.

Tanner, C.J., 2007. A case study for the Ecovillage at Currumbin - integrated water management planning, design and construction. In Water Resources Management IV. pp. 33-41.

Thakadu, O.T., 2005. Success factors in community based natural resources management in northern Botswana: Lessons from practice. Natural Resources Forum, 29(3), pp.199-212. Available at: http://doi.wiley.com/10.1111/j.1477-8947.2005.00130.x.

Tinsley, S. \& George, H., 2006. Ecological footprint of the Findhorn foundation and community. Sustainable Development Research ... Available at: http://www.ecovillagefindhorn.com/docs/FF Footprint.pdf.

Tukker, A., 2000. Life cycle assessment as a tool in environmental impact assessment. Environmental Impact Assessment Review, 20(4), pp.435-456.

Uwasu, M. et al., 2009. Educational initiative of Osaka University in sustainability science: Mobilizing science and technology towards sustainability. Sustainability Science, 4(1), pp.45-53.

Wackernagel, M. \& Rees, W., 1996. Our Ecological Footprint: Reducing Human Impact on the Earth, Available at: http://www.worldcat.org/isbn/9780865713123.

Warner, K., 2002. Linking Local Sustainability Initiatives with Environmental Justice. Local Environment, 7(1), pp.35-47.

Weber, C.L. \& Matthews, H.S., 2008. Food-miles and the relative climate impacts of food choices in the United States. Environmental Science and Technology, 42(10), pp.3508-3513. 
While, A., Jonas, A.E.G. \& Gibbs, D., 2010. From sustainable development to carbon control: Eco-state restructuring and the politics of urban and regional development. Transactions of the Institute of British Geographers, 35(1), pp.76-93.

Wilson, G., 2010. Multifunctional "quality" and rural community resilience. Transactions of the Institute of British Geographers, 35(3), pp.364-381.

Yang, L., Zhou, J. \& Yan, X., 2010. Environmental strategies analysis of cohousing based on ecological footprint. In 2010 International Conference on Mechanic Automation and Control Engineering, MACE2010. pp. 4314-4317. 